\title{
Seawater Short-Range Electromagnetic Wave Communication Method Based on OFDM Subcarrier Allocation
}

\author{
Jun Wang, Shilian Wang \\ College of Electronic Science and Technology, National University of Defense Technology, Changsha, China \\ Email: 13776201189@163.com
}

How to cite this paper: Wang, J. and Wang, S.L. (2019) Seawater Short-Range Electromagnetic Wave Communication Method Based on OFDM Subcarrier Allocation. Journal of Computer and Communications, 7, 63-71.

https://doi.org/10.4236/jcc.2019.710006

Received: July 22, 2019

Accepted: October 9, 2019

Published: October 16, 2019

\begin{abstract}
Aiming at the problems of underwater acoustic communication such as narrowband, low speed and multipath effect, this paper studies the high-speed communication technology of electromagnetic wave in short distance at sea. The channel model of seawater electromagnetic wave is given, and the performance of different modulation modes in this channel is analyzed. A method of seawater short-range high-speed electromagnetic wave communication based on OFDM is proposed. In order to reduce the BER of the system, a method of allocating different modulation modes to subcarriers in seawater channel is introduced, which combines frequency domain equalization. This method has low complexity, improves the utilization of frequency band, and improves the reliability. It can realize high-speed and reliable communication in short distance in seawater.
\end{abstract}

\section{Keywords}

Underwater Electromagnetic Wave, OFDM, Subcarrier Modulation

\section{Introduction}

Most underwater communications use acoustic waves as physical transmission media [1]. However, there are some disadvantages of acoustic wave, such as slow speed, narrow bandwidth, obvious multipath effect, and great influence of water environment. Electromagnetic wave has higher bandwidth, faster speed and less impact on natural environment in short distance underwater.

The underwater communication of electromagnetic wave is mainly limited by the drastic attenuation of water channel, which is a special constant parameter channel. For certain waters, the electromagnetic parameters are almost un- 
changed. The attenuation of electromagnetic wave is related to frequency and propagation distance. It belongs to time-invariant frequency selective fading channel in a certain period of time. For this channel, OFDM technology can be used to reduce the transmission rate on each subcarrier, and each subchannel is approximately flat. Combined with frequency domain equalization, the attenuation caused by sea water channel can be reduced. In addition, choosing different modulation modes for different subcarriers can improve the bandwidth utilization, reduce the BER of the system and improve the reliability of transmission. In this paper, a subcarrier allocation modulation scheme for seawater electromagnetic channel is studied and designed, which can realize short-distance high-speed electromagnetic wave communication in seawater.

\section{Channel Model of Seawater Electromagnetic Wave}

Water has a high relative dielectric constant and conductivity, which results in the propagation characteristics of electromagnetic wave in water are different from those in air, and the most notable performance is a large attenuation. Because there is only difference in conductivity between seawater and freshwater, this paper only studies the transmission channel of electromagnetic wave in seawater, and only needs to change the conductivity in freshwater channel.

\subsection{Attenuation Constants and Phase Constants of Electromagnetic Waves in Seawater}

The template is used to format your paper and style the text. All margins, column widths, line spaces, and text fonts are prescribed; please do not alter them. You may note peculiarities. For example, the head margin in this template measures proportionately more than is customary. This measurement and others are deliberate, using specifications that anticipate your paper as one part of the entire journals, and not as an independent document. Please do not revise any of the current designations.

The propagation constant of electromagnetic wave in sea water can be expressed as [2]

$$
\gamma=\omega \sqrt{j \omega \mu(\sigma+j \omega \varepsilon)}=\alpha+j \beta
$$

where $\omega=2 \pi f, f$ is the propagation frequency of electromagnetic wave, $\varepsilon=\varepsilon_{0} \varepsilon_{r}$ is the dielectric constant, $\varepsilon_{0}=1 /(36 \pi) \times 10^{-9} \mathrm{~F} / \mathrm{m}$ is the vacuum dielectric constant, $c$ is the speed of light, $\varepsilon_{r}$ is the relative dielectric constant, $\mu=\mu_{0} \mu_{r}$ is the permeability, $\mu_{0}=4 \pi \times 10^{-7} \mathrm{H} / \mathrm{m}$ is vacuum permeability, $\sigma$ is the conductivity. The complex propagation constant $\gamma$ is composed of attenuation constant $\alpha$ and phase constant $\beta$, and the expression can be obtained as follows.

$$
\alpha=\sqrt{\frac{\omega^{2} \mu \varepsilon}{2}} \sqrt{\sqrt{1+\left(\frac{\sigma}{\omega \varepsilon}\right)^{2}}-1}
$$




$$
\beta=\sqrt{\frac{\omega^{2} \mu \varepsilon}{2}} \sqrt{\sqrt{1+\left(\frac{\sigma}{\omega \varepsilon}\right)^{2}}+1}
$$

According to the propagation characteristics of electromagnetic wave in conductive medium, when $\sigma / \omega \varepsilon \ll 1$, the medium presents the characteristics of medium, and when $\sigma / \omega \varepsilon \gg 1$, the medium presents the characteristics of conductor. For seawater, the conductivity is usually $\sigma=4 \mathrm{~S} / \mathrm{m}$ and the dielectric constant $\varepsilon=81 \varepsilon_{0}$. When the frequency satisfies $f \ll 887 \mathrm{MHz}$, the seawater is a good conductor. The attenuation constant and the phase constant are approximately equal. Equations (2) and (3) can be simplified as follows.

$$
\alpha=\beta \approx \sqrt{\pi f \mu \sigma}
$$

It can be seen that the attenuation constant and phase constant of electromagnetic wave in sea water are related to frequency, and increase with the increase of frequency, so the lower frequency is preferred.

\subsection{Wavelength of Electromagnetic Wave in Seawater}

The wave length of electromagnetic wave in sea water is

$$
\lambda=\frac{2 \pi}{\beta}=2 \sqrt{\frac{\pi}{f \mu \sigma}}
$$

The wavelength of electromagnetic wave is also related to frequency. With the increase of frequency, the wavelength decreases gradually. For $100 \mathrm{KHz}$ electromagnetic wave, the wave length in air is $3000 \mathrm{~m}$, while in sea water it is only $5 \mathrm{~m}$. At the same frequency, the size of the antenna is much smaller than that of the air because the wavelength of the electromagnetic wave in sea water is very small. From this point of view, underwater electromagnetic wave communication is suitable for the use of small antennas, which is very beneficial for practical application.

\subsection{The Propagation Velocity of Electromagnetic Wave in Seawater}

The propagation velocity (phase velocity) of electromagnetic wave in seawater is

$$
v_{p}=\frac{\omega}{\beta} \approx 2 \sqrt{\frac{\pi f}{\mu \sigma}}
$$

The propagation velocity of electromagnetic wave increases with the increase of frequency. For $100 \mathrm{KHz}$ frequencies, the propagation speed in seawater is $5 \times$ $10^{5} \mathrm{~m} / \mathrm{s}$. Although the velocity is less than that in air, it is still more than 300 times faster than that in sea water (about $1500 \mathrm{~m} / \mathrm{s}$ ). So underwater electromagnetic wave communication has less time delay in information transmission and can ensure real-time performance.

\subsection{Channel Function of Seawater Electromagnetic Channel}

High conductivity and dielectric constant of sea water will lead to short circuit 
when metal antenna contacts with sea water directly, which makes it difficult to match the impedance between antenna and sea water. Therefore, ring antenna is usually used in underwater communication [3]. In order to transmit effectively, insulating protective cover must be used on the surface, such as Teflon [4].

The main modes of electromagnetic wave propagation in water are direct radiation, reflection and interface propagation. For short-range electromagnetic wave communication in seawater, it can be considered that there is only a direct path, and the path loss includes the absorption loss of seawater to electromagnetic wave and the spatial expansion loss of electromagnetic wave propagation.

According to Friis transmission formula [5], the receiving power can be expressed as

$$
P_{r}=\left(\frac{\lambda}{4 \pi d}\right)^{2} P_{t} G_{T} G_{R}|A|^{2}
$$

where $P_{t}$ is the transmitting power, $P_{r}$ is the receiving power, $G_{t}$ and $G_{r}$ are the gain of transmitting antenna and receiving antenna respectively, $A=|E| /\left|E_{0}\right|$ is the attenuation factor, which is equal to the ratio of the actual field strength of receiving point to the field strength of free space. If the electromagnetic wave propagates in the direction of $+\mathrm{Z}$, then $E=E_{0} e^{-\alpha z-j \beta z}$.

$$
[A]_{d B}=20 \lg e^{-\alpha z}=-20 \alpha z \lg e
$$

The ratio of input power of transmitting antenna to output power of receiving antenna is defined as underwater transmission loss $L$, which is expressed in decibels. Without considering the influence of antenna equipment, that is $\left[G_{T}\right]_{d B}=\left[G_{R}\right]_{d B}=0$, it reflects the power transmission of channel, that is, underwater path loss.

$$
L_{P}=10 \lg \left\{\left(\frac{4 \pi d}{\lambda|A|}\right)^{2} \times \frac{1}{G_{T} G_{R}}\right\}=20 \lg \left(\frac{4 \pi d}{\lambda}\right)-[A]_{d B}
$$

Considering that the underwater short-range electromagnetic wave communication is the mode of LOS propagation, $z=d$ in Formula (8) denotes the propagation distance. By substituting Formulas (5) and (8) into (9), the expression of path loss is obtained.

$$
L_{p}=6.021+8.686 \alpha d+20 \lg (\beta d)
$$

Therefore, the channel function of seawater electromagnetic wave channel is expressed as follows.

$$
H(f)=\frac{1}{2 d \beta(f)} e^{-d \alpha(f)-j d \beta(f)}
$$

It can be seen that the channel function is frequency dependent, so the seawater electromagnetic wave channel belongs to frequency selective fading channel.

Figure 1 and Figure 2 show the amplitude-frequency response and phase-frequency response of the channel at different distances, respectively. With the increase of frequency, the attenuation increases gradually, and the farther the distance is, the more serious the attenuation is and the greater the phase change is. 


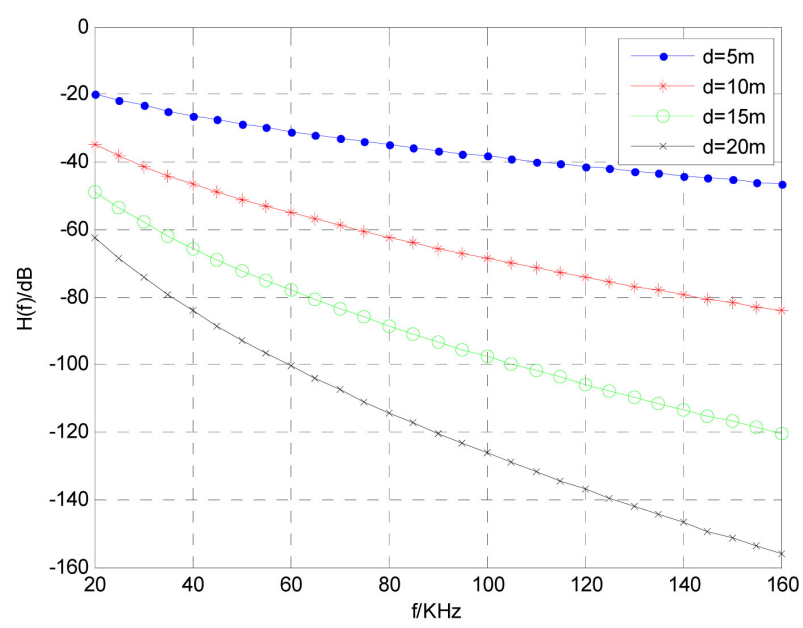

Figure 1. Amplitude-frequency response.

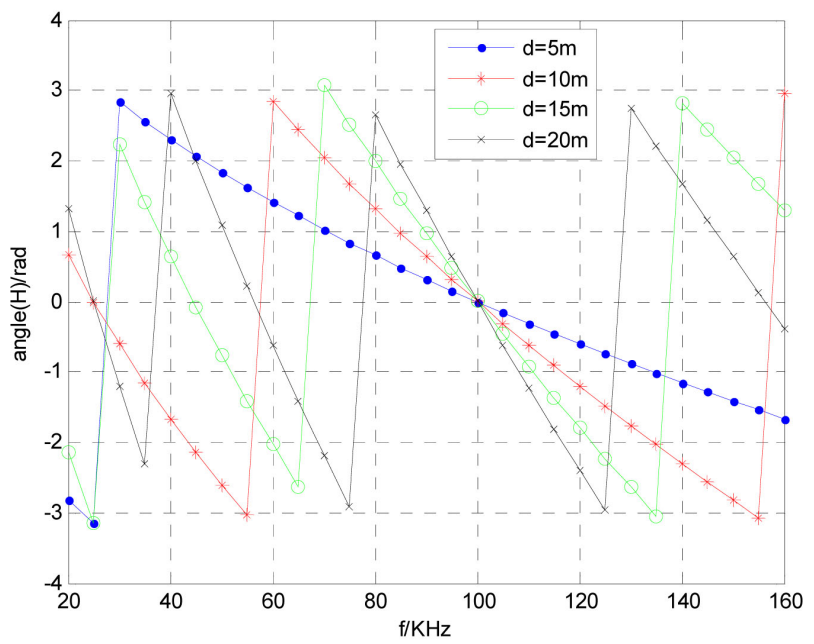

Figure 2. Phase-frequency response.

\section{Communication System and Its Parameter Selection}

We can see that the channel function of seawater electromagnetic wave decreases exponentially with frequency, which will lead to huge energy attenuation. Therefore, we can consider using OFDM communication system. Each subcarrier can be regarded as an independent subchannel. Because the data transmission rate on each subchannel is low, when the signal passes through the seawater electromagnetic channel, although there is fading in the whole signal frequency band, it can be approximately regarded as flat in each subchannel. Simple frequency domain equalization can eliminate the influence of channel. In addition, OFDM can greatly improve the spectral efficiency [6] [7], which is very suitable for the narrow bandwidth of seawater electromagnetic channel.

This paper chooses the communication distance of 15 meters, the center carrier frequency is $110 \mathrm{KHz}$, the bandwidth is $20 \mathrm{KHz}$, and adopts five kinds of modulation methods: BPSK, QPSK, 8PSK, 16QAM and 64QAM. The number of OFDM subcarriers is 64 , and the receiving end adopts ZF equalization. 


\section{Simulation Analysis}

The BER of receiver is simulated under five modulation modes: single carrier BPSK, QPSK, 8PSK, 16QAM and 64QAM. The carrier frequency is $110 \mathrm{KHz}$, the communication distance is $15 \mathrm{~m}$, and the bandwidth is $20 \mathrm{KHz}$.

Figure 3 shows the BER of the receiver after ZF equalization under five modulation modes. It can be seen that BPSK and QPSK have the same BER, and their performance is much better than that of 8PSK, 16QAM and 64QAM.

According to the previous research and Figure 4, it can be seen that many high frequency subcarriers of OFDM signal fade seriously after passing through the sea water electromagnetic channel, so that the BER is very high. Because of the small attenuation in low frequency band and the large attenuation in high frequency band, the BER of low order modulation is lower under the same power. Therefore, we consider a modulation method that assigns different subchannels. High-order modulation is used for low-frequency subcarriers and low-order modulation is used for high-frequency subcarriers, so as to achieve the goal of considering both error performance and spectrum utilization.

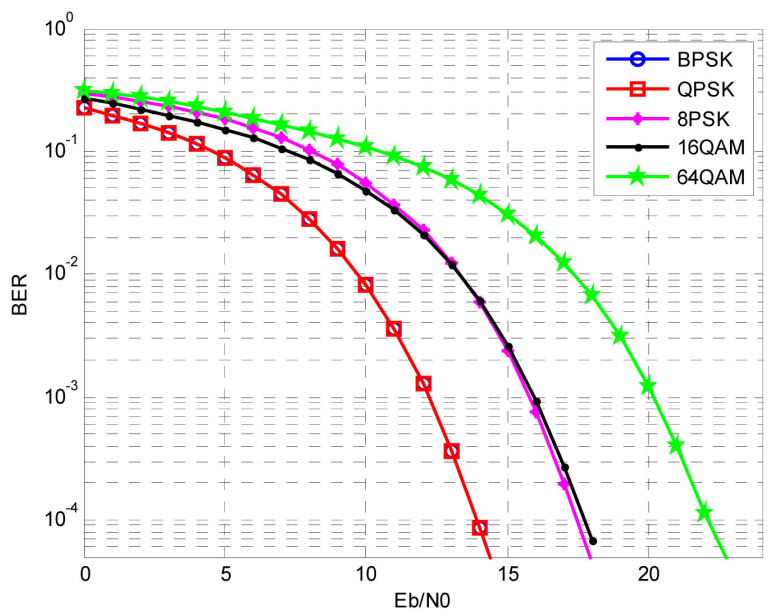

Figure 3. BER comparison of five modulation modes.

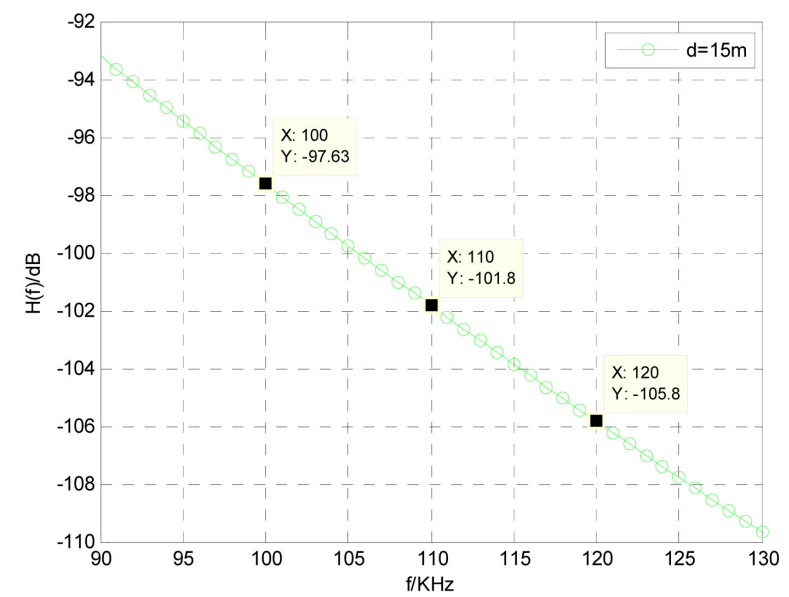

Figure 4. Amplitude-frequency response at $15 \mathrm{~m}$ distance. 
Table 1 lists the Eb/N0 required for the five modulation modes to propagate $15 \mathrm{~m}$ in the seawater electromagnetic channel with BER $=10^{-4}$. Combining with the amplitude-frequency response at $15 \mathrm{~m}$ propagation distance, it can be observed that the difference between the $\mathrm{Eb} / \mathrm{N} 0$ required by $\mathrm{BER}=10^{-4}$ in three modes of QPSK, 16QAM and 64QAM is approximately equal to the difference between the amplitude response of $120 \mathrm{~K}, 110 \mathrm{~K}$ and $100 \mathrm{~K}$.

In addition, by observing the amplitude-frequency response, it can be seen that $\mathrm{H}(\mathrm{f}) / \mathrm{dB}$ basically decreases linearly in the range of 100 to $120 \mathrm{KHz}$. According to this phenomenon, an optimized subcarrier allocation scheme can be considered. For the attenuation near $120 \mathrm{KHz}$, QPSK modulation is adopted, $110 \mathrm{KHz}$ adopts 16QAM, and the attenuation of $100 \mathrm{KHz}$ adopts 64QAM, and the three modes are evenly divided in the bandwidth. For ease of implementation, the 64 subcarriers in the 100 to $120 \mathrm{kHz}$ bandwidth are approximately equally divided into three groups, namely, 21 64QAM, 21 16QAM, and 22 QPSK.

The other conditions remain unchanged. The simulation results based on the optimization scheme are shown in Figure 5.

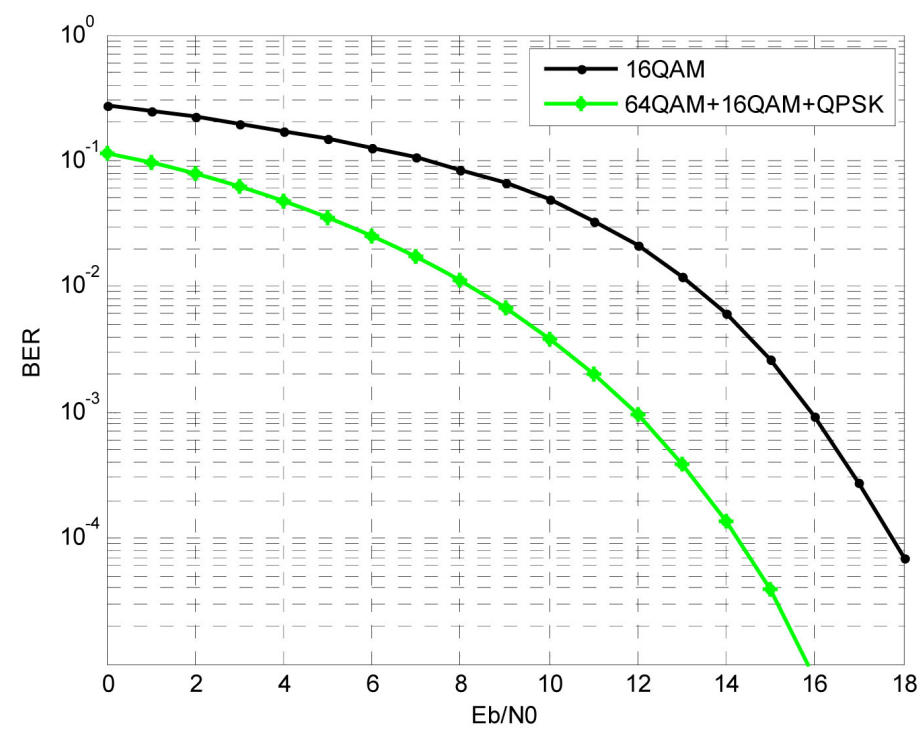

Figure 5. BER comparison between optimized scheme and 16QAM.

Table 1. Eb/N0 of five modulation modes and $\mathrm{H}(\mathrm{f})$.

\begin{tabular}{ccccc}
\hline Modulation mode & BPSK/QPSK & 8PSK & 16QAM & 64QAM \\
\hline Eb/N0 for BER $=10^{-4}$ & $13.9 \mathrm{~dB}$ & $17.5 \mathrm{~dB}$ & $17.8 \mathrm{~dB}$ & $22.1 \mathrm{~dB}$ \\
Difference & $3.6 \mathrm{~dB}$ & $0.3 \mathrm{~dB}$ & $4.3 \mathrm{~dB}$ \\
Frequency & $120 \mathrm{KHz}$ & $110 \mathrm{KHz}$ & $100 \mathrm{KHz}$ \\
H (f) & $-105.8 \mathrm{~dB}$ & $-101.8 \mathrm{~dB}$ & $-97.6 \mathrm{~dB}$ \\
Difference & \multicolumn{2}{c}{$4 \mathrm{~dB}$} & $4.2 \mathrm{~dB}$ \\
\hline
\end{tabular}


From the simulation results, it can be seen that the BER of the optimized subcarrier allocation is lower than that of 16QAM. Taking the BER $=10^{-4}$ as an example, the required $\mathrm{Eb} / \mathrm{N} 0$ is reduced by about $3.2 \mathrm{~dB}$. Because the ratio of modulation order of the three modulation modes is $6: 4: 2$, the bandwidth utilization of the optimized scheme is the same as that of 16QAM. It can be seen that the optimization scheme can take into account both the bandwidth utilization and the reliability.

\section{Conclusion}

Seawater electromagnetic wave channel is a special frequency selective fading channel. The attenuation of signal amplitude and phase change are greatly affected by frequency and transmission distance. In this paper, channel modeling is carried out through theoretical derivation, and simulation shows that QPSK is the optimal modulation mode for single carrier. Combining the performance of five modulation modes and channel amplitude-frequency characteristics under single carrier, a communication scheme using OFDM system and assigning different modulation modes to subcarriers is proposed. The simulation results show that its performance is improved by about $3.2 \mathrm{~dB}$ compared with 16QAM. This scheme can achieve the bandwidth utilization of 16QAM and reduce BER at the same time. Compared with the single QPSK mode, the transmission power is increased to a certain extent, but the information rate can be increased by two times, and the bandwidth utilization can be improved. More information can be transmitted in the narrow bandwidth marine electromagnetic channel.

\section{Conflicts of Interest}

The authors declare no conflicts of interest regarding the publication of this paper.

\section{References}

[1] Che, X., Wells, I., Dickers, G., et al. (2010) Re-Evaluation of RF Electromagnetic Communication in Underwater Sensor Networks. IEEE Communications Magazine, 48, 143-151. https://doi.org/10.1109/mcom.2010.5673085

[2] Hunt, K.P., Niemeier, J.J. and Kruger, A. (2010) RF Communications in Underwater Wireless Sensor Networks. IEEE International Conference on Electro/Information Technology. https://doi.org/10.1109/eit.2010.5612087

[3] Al-Shamma'A, A.I., Shaw, A. and Saman, S. (2004) Propagation of Electromagnetic Waves at $\mathrm{MHz}$ Frequencies Through Seawater. IEEE Transactions on Antennas \& Propagation, 52, 2843-2849. https://doi.org/10.1109/tap.2004.834449

[4] Siegel, M. and King, R. (1973) Electromagnetic Propagation between Antennas Submerged in the Ocean. IEEE Transactions on Antennas and Propagation, 21, 507-513. https://doi.org/10.1109/tap.1973.1140525

[5] Guru, B.S., Hiziroglu and Hüseyin, R. (2005) Electromagnetic Field Theory Fundamentals: Electromagnetic Field Theory. China Machine Press. (In Chinese) https://doi.org/10.1017/cbo9781139165297.002

[6] Kelley, B., Manoj, K. and Jamshidi, M. (2009) Broadband RF Communications in 
Underwater Environments Using Multi-Carrier Modulation. IEEE International Conference on Systems. IEEE Press. https://doi.org/10.1109/icsmc.2009.5345980

[7] Kelley, B. and Naishadham, K. (2013) RF Multicarrier Signaling and Antenna Systems for Low SNR Broadband Underwater Communications. IEEE Radio \& Wireless Symposium. https://doi.org/10.1109/rws.2013.6486734 